Cite this: Phys. Chem. Chem. Phys., 2013.

\title{
LSPR properties of metal nanoparticles adsorbed at a liquid-liquid interface $\dagger$
}

15, 5374

Received 18th November 2012,

Accepted 7th January 2013

DOI: $10.1039 / c 3 c p 44101 f$

www.rsc.org/pccp

\author{
Zhilin Yang, ${ }^{\star a}$ Shu Chen, ${ }^{\mathrm{a}}$ Pingping Fang, ${ }^{\mathrm{b}}$ Bin Ren, ${ }^{\mathrm{c}}$ Hubert H. Girault ${ }^{\star \mathrm{b}}$ and \\ Zhongqun Tian*c
}

\begin{abstract}
Unlike the solid-air and solid-liquid interfaces, the optical properties of metal nanoparticles adsorbed at the liquid-liquid interface have not been theoretically exploited to date. In this work, the three dimensional finite difference time domain (3D-FDTD) method is employed to clarify the localized surface plasmon resonance (LSPR) based optical properties of gold nanoparticles (NPS) adsorbed at the water-oil interface, including near field distribution, far field absorption and their relevance. The LSPR spectra of NPs located at a liquid-liquid interface are shown to differ significantly from those in a uniform liquid environment or at the other interfaces. The absorption spectra exhibit two distinct LSPR peaks, the positions and relative strengths of which are sensitive to the dielectric properties of each liquid and the exact positions of the NPs with respect to the interface. Precise control of the particles' position and selection of the appropriate wavelength of the excitation laser facilitates the rational design and selective excitation of localized plasmon modes for interfacial NPs, a necessary advance for the exploration of liquid-liquid interfaces via surface enhanced Raman spectroscopy (SERS). According to our calculations, the SERS enhancement factor for Au nanosphere dimers at the water-oil interface can be as high as $10^{7}-10^{9}$, implying significant promise for future investigations of interfacial structure and applications of liquid-liquid interfaces towards chemical analysis.
\end{abstract}

\section{Introduction}

Liquid-liquid interfaces are as important as solid-liquid, air-liquid or air-solid interfaces in modern science and technology. ${ }^{1-14}$ The interfacial structure of a liquid is very different from that of the bulk liquid because the molecules in the liquid phase are by definition mobile. ${ }^{15}$ However, unlike the other interfaces, the liquid-liquid interface has not been investigated sufficiently.

Excellent contributions regarding chemical reactions at liquid interfaces by Benjamin ${ }^{16}$ and ion-distributions near a liquid-liquid interface by Schlossmann and co-workers ${ }^{2}$ have

\footnotetext{
${ }^{a}$ Department of Physics, Xiamen University, Xiamen 361005, China. E-mail: zlyang@xmu.edu.cn; Tel: +86-592-2188510

${ }^{b}$ Laboratoire d'Electrochimie Physique et Analytique, Ecole Polytechnique Fédérale de Lausanne, Station 6, CH-1015 Lausanne, Switzerland. E-mail: hubert.girault@epfl.ch

${ }^{c}$ State Key Laboratory of Physical Chemistry of Solid Surfaces and Department of Chemistry, College of Chemistry and Chemical Engineering, Xiamen University, Xiamen 361005, China. E-mail: zqtian@xmu.edu.cn; Tel: +86-592-2186979 $\dagger$ Electronic supplementary information (ESI) available: The calculation model (Fig. S1) and coresponding SERS enhancement factor at the hot spot under vertical incidence for gold nanoparticle dimers (Fig. S2). See DOI: 10.1039/c3cp44101f
}

added significantly to the development of our understanding of the liquid-liquid interface. In general, optical and nonlinear optical spectroscopic techniques such as Raman spectroscopy, ${ }^{17}$ infrared spectroscopy, ${ }^{18}$ UV-vis spectroscopy, ${ }^{19}$ second harmonic generation $^{1,4}$ and sum-frequency generation ${ }^{3,4,12,20}$ together with X-ray diffraction ${ }^{1,2,18}$ and atomic force microscopy, ${ }^{21}$ may be applied experimentally to probe the interfacial structure of a liquid. The physical and chemical properties of a liquid interface are directly related to its interfacial structure. Raman spectroscopy provides fingerprint information to fully characterize interfacial species, offering a powerful way to explore the structure and reaction pathways at liquid-liquid interfaces. However, normal spontaneous Raman scattering is intrinsically very weak and often signals of sufficient quality for structural information analysis from the interface are difficult to attain unless extra enhancements are provided. Fortunately, the interface between two immiscible liquids has been confirmed as an important means of generating two dimensional self-assemblies of metal nanoparticles, ${ }^{6-9,22-27}$ and localized plasmon resonance (LSPR) has been experimentally observed for a planar close-packed assembly of gold nanoparticles adsorbed at the water-1,2-dichloroethane interface. ${ }^{11}$ These metal nanoparticles, with suitable LSPR bands, are capable of enhancing 
the efficiencies of Raman scattering by as much as $10^{6}-10^{10}$ fold if the exciting laser has an appropriate wavelength. Such a phenomenon, widely known as surface-enhanced Raman spectroscopy (SERS), ${ }^{28,29}$ offers an enormous enhancement in signal allowing sensitive spectroscopic detection and even identification of single molecules located on the nanoparticle surface. Thus, the primary disadvantage of conventional Raman may be overcome by the assembly of metal nanoparticles at the liquid-liquid interface. However, to the best of our knowledge, systematic investigations of the LSPR modes and corresponding SERS enhancement of nanoparticles at liquid-liquid interfaces have not been well established. Herein, we present the theoretical LSPR-based optical properties and SERS properties of gold nanoparticles at the water-oil interface based on 3D-FDTD numerical simulations, including far field and near field features.

\section{Calculation method}

The FDTD approach has become one of the most important computational methods in electromagnetics since it was proposed by Yee in $1966 .{ }^{30}$ The FDTD method involves the discretization of Maxwell's equations in both the time and the space domain in order to find the $\mathrm{E}$ and $\mathrm{H}$ fields at different positions and at different time-steps. This method can conveniently be applied to simulate the electromagnetic scattering and radiation from a target of complex shape as well as nonuniform dielectric objects by simply adjusting the number, size and material properties of the Yee cell. ${ }^{31,32}$ All calculations in this work were performed with commercial Lumerical FDTD solutions (version 7.5) software. Details of the calculation method can be found elsewhere. ${ }^{33}$

The calculation model is illustrated in Fig. 1. Water and oil are selected as two typical immiscible liquids capable of forming an ideal liquid-liquid interface. Single gold nanosphere and nanosphere dimers were selected as the interfacial objects to be probed during the present LSPR study. Free manipulation of their positions relative to the interface is assumed, regardless of the current limited availability of experimental methods to achieve such precise spatial control. The incident plane wave propagated along the $y$-axis direction

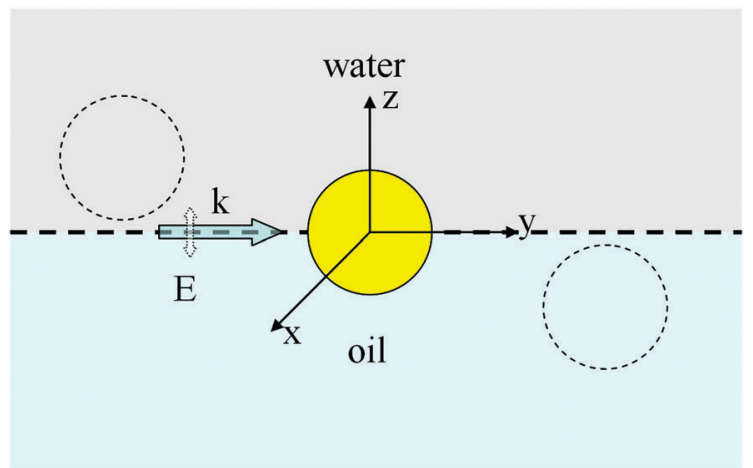

Fig. 1 Schematic of Au nanoparticles at a water-oil interface with diameter $d=20 \mathrm{~nm}$. with the polarization along the $z$-direction. Perfectly matched layer (PML) boundary conditions were used in the FDTD simulation. The simulation time in all calculations is set to $1000 \mathrm{fs}$, which is long enough to ensure calculation convergence. In general, the smaller the Yee cell size, the better the calculation results. However, due to the limitation of computation resources, cell size cannot be infinitely small for practical purposes. The Yee cell size in this work is set to $0.25 \times 0.25 \times$ $0.25 \mathrm{~nm}^{3}$ in all cases. The frequency dependent optical constant of $\mathrm{Au}$ is directly taken from Johnson and Christy, ${ }^{34}$ and the dielectric constants of water and oil are 1.77 and 5.0 respectively. The latter value has been chosen especially to be very large to highlight the influence of the dielectric discontinuity on the LSPR response. The diameter of Au nanospheres is set at 20 nanometers.

\section{Results and discussion}

The unique optical properties of metallic nanoparticles are mainly due to their localized surface plasmon resonance, which involves the coherent oscillation of free conduction band electrons in resonance with an incident electromagnetic field. For nanospheres submerged into a homogeneous liquid, such as water or oil, only one LSPR peak is observed in the absorption spectra (523 nm for water, $622 \mathrm{~nm}$ for oil), as shown in Fig. 2. Such an observation for metal nanoparticles (NPs) in a uniform liquid dielectric environment has been widely investigated and the related theory was successfully developed in the past century. ${ }^{35}$ However, from Fig. 2, one can see that the absorption spectra of Au NPs located at the water-oil interface are quite different from those in a uniform water or oil environment. Two distinct peaks, located at about $535 \mathrm{~nm}$ and $580 \mathrm{~nm}$ respectively, appear in the absorption spectra rather than a single LSPR peak. These two peaks imply the simultaneous existence of two distinct LSPR modes for $\mathrm{Au}$ particles at a water-oil interface under white light excitation. Theoretically, the resonance of localized surface plasmon modes in the water $(523 \mathrm{~nm})$ and oil $(622 \mathrm{~nm})$, respectively, may generate the two peaks that are clearly identifiable by the calculated SERS electromagnetic (EM) enhancement distribution on the $y z$-plane, as shown in Fig. 3. With regard to SERS applications at the interface, discussed later, the near field enhancement distribution

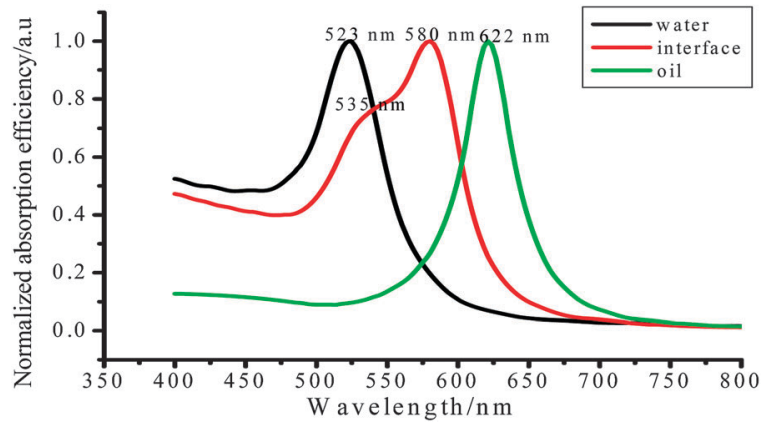

Fig. 2 Calculated optical absorption spectra (far field) for a gold nanoparticle in water (black line), oil (green line), and at the water-oil interface (red line). 

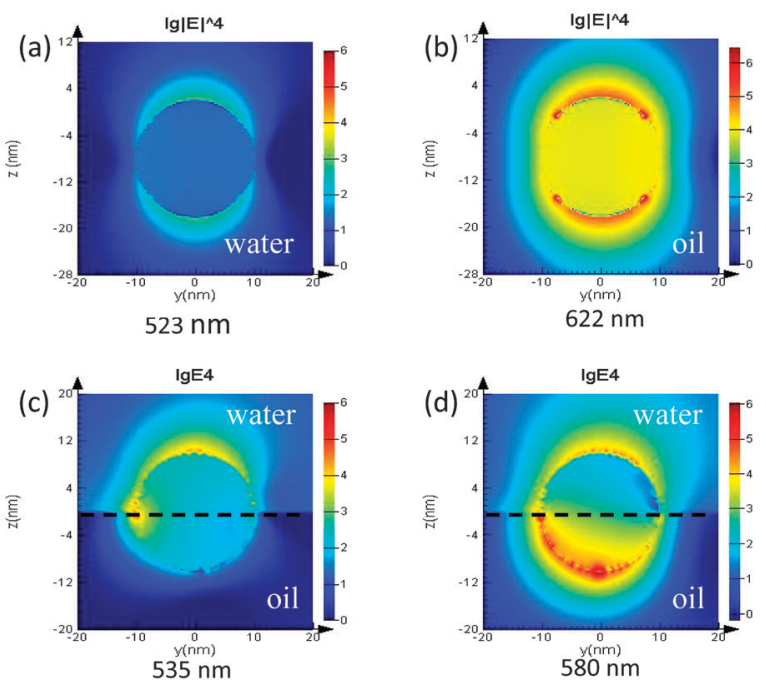

Fig. 3 FDTD calculated electromagnetic enhancement distribution (near field) for SERS in the $y z$-plane under the surface plasmon resonance conditions. Dashed line represents the water-oil interface.

(defined as $M=\frac{E_{\mathrm{loc}}}{E_{\mathrm{in}}}$ where $E_{\mathrm{loc}}$ and $E_{\mathrm{in}}$ are the magnitudes of the localized and incident fields, respectively) was replaced by the common logarithm of the EM enhancement of SERS $\left(\approx M^{4}\right)$, from which one can determine the order of the maximum SERS EM enhancement factor and the location of the hot spot directly. As shown in Fig. 3(a) and (b), for a Au nanoparticle immersed in a uniform liquid environment, the resultant LSPR near field distribution is highly symmetrical and the plasmon mode arises from the resonance of the dipole mode. However, for an identical Au nanoparticle at the liquid-liquid interface, the symmetry of the near field distribution was broken (Fig. 3(c) and (d)). Upon laser excitation at $535 \mathrm{~nm}$, corresponding to the first LSPR peak position in the absorption spectra, the enhanced near field region is mainly concentrated at the particle's surface on the aqueous side of the interface. Since this LSPR peak is just slightly red-shifted with respect to the absorption spectrum in water, and significant field enhancement can be observed at the nanoparticle-water interface, we assign this LSPR mode as a "Water mode". Similarly, upon laser excitation at $580 \mathrm{~nm}$ (the second peak position, blueshifted with respect to the absorption spectrum in oil), LSPR of the Au nanoparticle at the water-oil interface is mainly decided by the LSPR mode in oil. Accordingly, the field enhancement region transfers to the particle-oil interface and this LSPR mode is defined as an "Oil mode".

The two different LSPR peaks and the corresponding near field distribution modes for gold nanospheres at the water-oil interface in Fig. 2 and 3 imply a strong correlation between the far field and near field. To elucidate the intrinsic principle and quantitatively reveal the relationship between the far field and the near field in such a system, the dependence of the absorption efficiency and the field intensity enhancement at two typical points (A and B, schematically illustrated in Fig. 4) on the excitation wavelength were calculated. The different influences of LSPR

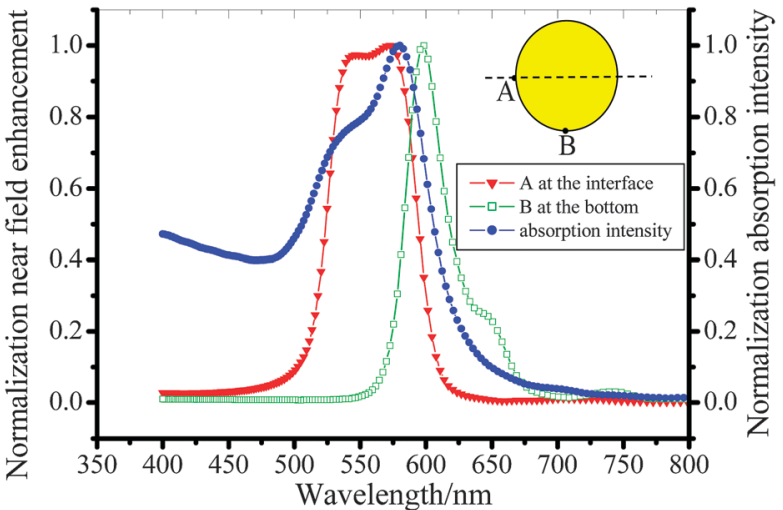

Fig. 4 Electric field intensity enhancement of two points ( $A$ is located at the water-oil interface, $B$ is a point at the bottom of the nanoparticle in oil) and absorption intensity over the spectral range $400-800 \mathrm{~nm}$. The three curves in the figure have been normalized. The spherical center of the Au nanoparticle is located at the water-oil interface.

on the far and near fields can be found in Fig. 4. For point A, analogous to the absorption spectra of a nanoparticle located at the water-oil interface, two field enhancement peaks correspond to the water and oil modes, respectively. It should be noted that the value of the field enhancement factors at $530 \mathrm{~nm}$ to $575 \mathrm{~nm}$ are comparable, meaning the EM enhancements exhibit no detectable differences for the two LSPR modes. Unlike point A, only one field enhancement peak, the position of which is slightly red-shifted with respect to the absorption spectra, is observed for point $B$. The resonance frequency and field enhancement at point B is only dependent on the oil mode of the LSPR as this point is far away from the interface and the influence of the water mode may be neglected. The peak positions for the near-field enhancement at both point A and B were noted to deviate slightly from that in the absorption spectra. The origin of the deviation in peak position between the far field and near field may be attributed to two factors. First, the near-field enhancement mainly originates from LSPR resulting from the light scattering effect of nanoparticles, while the absorption spectra are directly related to the absorption of energy by Au nanoparticles. Second, the absorption is proportional to the polarizations of various multipoles, and higher order multipoles usually give a small contribution to the far field. However, for the near field, both the dipoles and higher order multipoles critically influence its electric field intensity. In fact, this deviation was also experimentally observed by the Van Duyne group. ${ }^{36}$ It should also be pointed out that when the mode corresponding to water or oil was excited, the property of the LSPR is slightly affected by the dielectric environment at the other part. On division of a Au nanoparticle into two equal parts by the water-oil interface, from Fig. (4), one can see that the higher dielectric constant of oil has a stronger influence on the water mode of LSPR than the water's influence on the oil's mode.

The discussion thusfar infers that the peak position and relative strengths of the LSPR peaks should be sensitive to the position of the nanoparticle relative to the interface. Precise control of a 

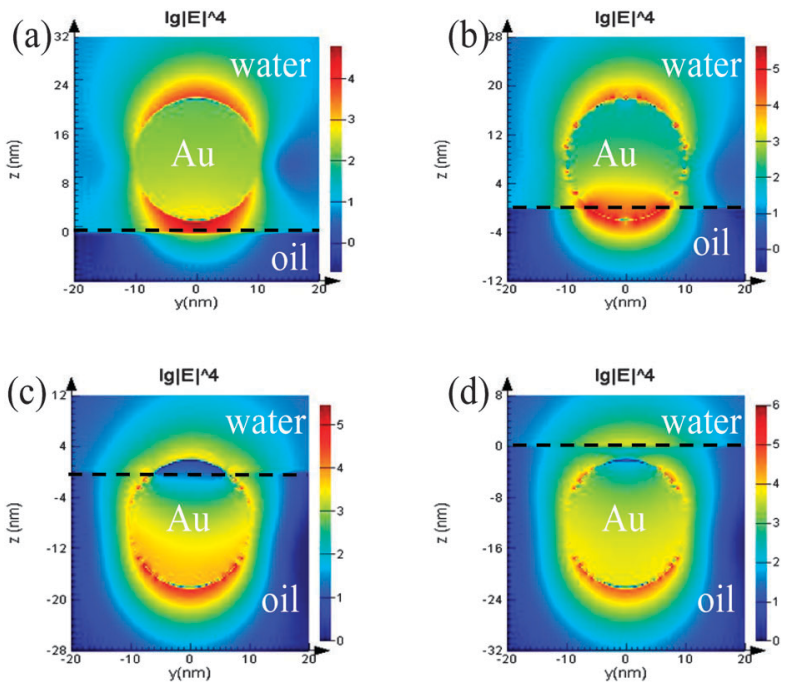

Fig. 5 Calculated SERS electromagnetic enhancement distribution in the $y z$-plane at the frequency of LSPR for various positions of Au nanoparticle. (a) $2 \mathrm{~nm}$ above the interface. (b) $2 \mathrm{~nm}$ submerged in oil. (c) $2 \mathrm{~nm}$ remaining in water. (d) $2 \mathrm{~nm}$ below the interface.

nanoparticle location relative to the interface may be manipulated experimentally by the nature of the liquid-liquid interface, surface modification of the nanoparticles and the effective radius of the nanoparticles. ${ }^{9,37}$ To determine whether the LSPR mode and field enhancement properties for $\mathrm{Au}$ nanoparticles are sensitive to their spatial positions relative to the interface, the maximum SERS electromagnetic enhancement distributions in the $y z$-plane for a series of $\mathrm{Au}$ nanosphere positions were calculated and graphically illustrated in Fig. 5. For simplicity, only four particle positions were selected in the simulation. When a Au nanoparticle approaches and passes through the interface moving from the water to the oil side, the maximum SERS EM enhancement at the hot spot becomes higher and higher. Although the hot spot is always located at the particle's surface, its specific location varies with the particle's position. Compared with the 4 orders of SERS enhancement in water (see Fig. 5(a)), the SERS enhancement at the hot spot increases to about 5 orders when the Au nanosphere is $2 \mathrm{~nm}$ away from the interface, as shown in Fig. 5(b). Clearly, the highly localized hot spot at the water-oil interface and the 4-5 orders of SERS enhancement offer us an ideal opportunity to investigate the behavior of molecules at the liquid-liquid interface with Raman spectroscopy. This technique is a reliable and relatively simple optical probe tool for studying a liquid-liquid system. If $\mathrm{Au}$ nanoparticles gradually approach the interface and immerse themselves on the oil side, the water mode of LSPR will decay while the oil mode becomes dominant. Although the maximum SERS enhancement at the hot spot under oil mode is close to 6 orders (see Fig. 5(d)), it seems to be of no remarkable significance for interfacial analysis as the location of the hot spot transfers from the interface to the portion of the particles' surface submerged on the oil side (see Fig. 5(c) and (d)).

To this point, herein, we have discussed the LSPR properties of an isolated gold nanoparticle absorbed at the liquid-liquid interface.
The next step is to investigate the plasmon resonance and EM enhancement arising from the interaction of two (or more) nanoparticle aggregates. SERS is often not a single particle effect. An efficient SERS-active system is typically strongly dependent on particle-particle or particle-substrate near field coupling effects and these coupling effects become particularly important for small inter-nanoparticle separation. ${ }^{38,39}$ Here, we make further efforts to apply the FDTD method to a nanoparticle dimer as a model system illustrating the most important characteristics of gap plasmon resonance for LSPR studies at a water-oil interface. Such a model is relevant as assemblies of $\mathrm{Au}$ nanoparticles at the liquid-liquid interface often adopt close-packed formations, as confirmed experimentally on numerous occasions. ${ }^{6,8,10,23,24}$ In the calculation, two identical $20 \mathrm{~nm} \mathrm{Au}$ nanospheres are separated by a gap of $1 \mathrm{~nm}$, their spatial locations set precisely at the interface, and the symmetric axis of the dimer is along the $y$-axis. The excitation laser is the same as that in Fig. 1. Calculated absorption spectra and near field distributions corresponding to the LSPR peaks in the absorption spectra are shown in Fig. 6. For nanoparticle dimers, in addition to the two LSPR modes (at $533 \mathrm{~nm}$ and $576 \mathrm{~nm}$ ) that arise from the excitation of the water and oil modes, respectively, another red-shifted LSPR peak (749 nm) appears in the absorption spectra (see the red line in Fig. 6). This new LSPR peak at $749 \mathrm{~nm}$ originates from the strong near field coupling effect, which is clearly identified by the enhanced field distribution (see the inset in Fig. 6). The maximum SERS enhancement factor at the gap is in excess of $10^{7}$. From the calculation results in Fig. 6, it should be noted that all three LSPR modes (water mode, oil mode and coupling mode) contribute effectively to SERS enhancement at the interface for dimers. This is in contrast to an isolated nanoparticle where the oil mode doesn't show any remarkable SERS enhancement at the liquid-liquid interface. Further calculations show that if the incident plane wave propagates along the $z$-axis with the polarization along the dimer-axis direction, the stronger near field coupling effect will result in a higher SERS enhancement factor of $10^{9}$ ! The calculation model and corresponding results can be found in Fig. S1 and S2 of the ESI. $†$

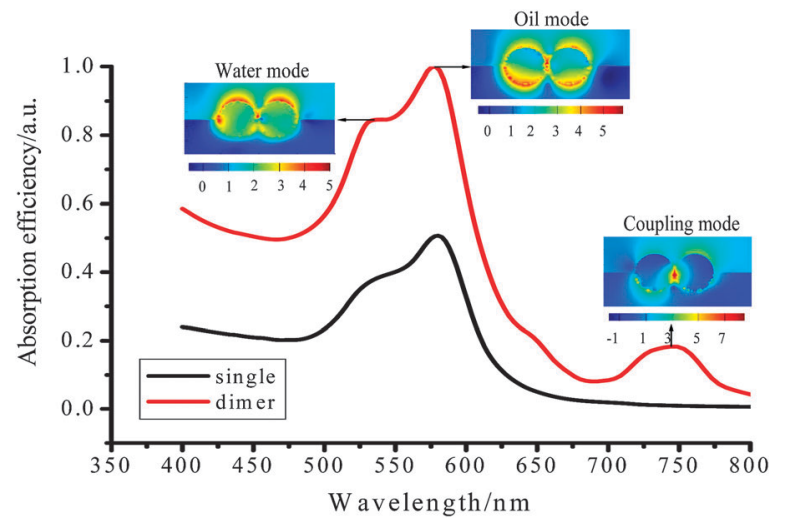

Fig. 6 Calculated absorption spectra of gold nanoparticle dimers (red line) at the water-oil interface and the corresponding electric field distribution at three LSPR peaks. 


\section{Conclusions}

In conclusion, a theoretical understanding of the localized surface plasmon resonance properties of an isolated Au nanoparticle and $\mathrm{Au}$ nanoparticle dimer at the water-oil interface has been developed for the first time. Two distinct LSPR peaks, corresponding to two different plasmon modes, arise in the far field absorption spectra. The position and relative strength of LSPR is found to be fundamentally dependent on the exact spatial position of the nanoparticle relative to the interface, and the different influences of LSPR on the far and near fields was given. The coupling effect dependence of LSPR for gold nanoparticle dimers adsorbed at a water-oil interface was also discussed. The calculated results reveal that the maximum SERS enhancement factor of the hot spot located at the interface can be as high as 7-9 orders of magnitude. This study provides a strong theoretical framework within which the potential applications of SERS at liquid-liquid interfaces, for interfacial structural analysis for example, may be developed.

\section{Acknowledgements}

This work was supported by the NSF of China (21173171 and 11074210), the Fundamental Research Funds for the Central Universities (2012121013) and the MOST grant No. 2009CB930703.

\section{Notes and references}

1 W. H. Steel and R. A. Walker, Nature, 2003, 424, 296.

2 G. M. Luo, S. Malkova, J. Yoon, D. G. Schultz, B. H. Lin, M. Meron, I. Benjamin, P. Vanýsek and M. L. Schlossman, Science, 2006, 311, 216.

3 P. B. Miranda and Y. R. Shen, J. Phys. Chem. B, 1999, 103, 3292.

4 M. Fedoseeva, S. Richert and E. Vauthey, Langmuir, 2012, 28, 11291.

5 B. Su, N. Eugster and H. H. Girault, J. Am. Chem. Soc., 2005, 127, 10760.

6 Y. Lin, H. Skaff, T. Emrick, A. D. Dinsmore and T. P. Russel, Science, 2003, 299, 226.

7 N. Bowden, A. Terfort, J. Carbeck and G. M. Whitesides, Science, 1997, 276, 233.

8 E. Glogowski, R. Tangirala, J. B. He, T. P. Russell and T. Emrick, Nano Lett., 2007, 7, 389.

9 W. H. Binder, Angew. Chem., Int. Ed., 2005, 44, 5172.

10 P. Arumugam, D. Patra, B. Samanta, S. S. Agasti, C. Subramani and V. M. Rotello, J. Am. Chem. Soc., 2008, 130, 10046.

11 M. Hojeij, N. Younan, L. Ribeaucourt and H. H. Girault, Nanoscale, 2010, 2, 1665.

12 D. Chandler, Nature, 2007, 445, 831.
13 D. Schaming, M. Hojeij, N. Younan, H. Nagatani, H. J. Lee and H. H. Girault, Phys. Chem. Chem. Phys., 2011, 13, 17704.

14 A. A. Kornyshev, M. Marinescu, J. Paget and M. Urbakh, Phys. Chem. Chem. Phys., 2012, 14, 1850.

15 X. Wang, Q. Peng and Y. D. Li, Acc. Chem. Res., 2007, 40, 635.

16 I. Benjamin, Chem. Rev., 1996, 96, 1449.

17 M. D. Serio, H. Mohapatra, R. Zenobi and V. Deckert, Chem. Phys. Lett., 2006, 417, 452.

18 M. Maas, H. Rehage, H. Nebel and M. Epple, Langmuir, 2009, 25, 2258.

19 I. Hatay, et al., J. Am. Chem. Soc., 2010, 132, 13733.

20 K. V. Nelson and I. Benjamin, J. Phys. Chem. B, 2012, 116, 4286.

21 D. Filip, V. I. Uricanu, M. H. G. Duits, W. G. M. Agterof and J. Mellema, Langmuir, 2005, 21, 115.

22 H. W. Duan, D. Y. Wang, N. S. Sobal, M. Giersig, D. G. Kurth and H. Möhwald, Nano Lett., 2005, 5, 949.

23 S. H. Chen, H. Yao and K. Kimura, Langmuir, 2001, 17, 733.

24 L. Isa, K. Kumar, M. Müller, J. Grolig, M. Textor and E. Reimhult, ACS Nano, 2010, 4, 5665.

25 M. E. Flatté, A. A. Kornyshev and M. Urbakh, J. Phys. Chem. C, 2012, 174, 1735.

26 L. H. Qian, S. J. Zhai, Y. T. Jiang and B. Das, J. Mater. Chem., 2012, 22, 4932.

27 M. T. Sun and H. X. Xu, Small, 2012, 8, 2777.

28 M. Moskovits, J. Raman Spectrosc., 2005, 36, 485.

29 P. L. Stiles, J. A. Dieringer, N. C. Shah and R. P. Van Duyne, Annu. Rev. Anal. Chem., 2008, 1, 601.

30 K. Yee, IEEE Trans. Antennas Propag., 1966, 14, 302.

31 C. Oubre and P. Nordlander, J. Phys. Chem. B, 2005, 109, 10042.

32 Z. L. Yang, Q. H. Li, B. Ren and Z. Q. Tian, Chem. Commun., 2011, 47, 3909.

33 K. S. Kunz and R. J. Luebber, The Finite Difference Time Domain Method for Electromagnetics, CRC press, Cleveland, 1993.

34 P. B. Johnson and R. W. Christy, Phys. Rev. B: Solid State, 1972, 6, 4370.

35 C. F. Bohren and D. R. Huffman, Absorption and Scattering of Light by Small Particles, Wiley Interscience, New York, 1983.

36 A. D. McFarland, M. A. Young, J. A. Dieringer and R. P. Van Duyne, J. Phys. Chem. B, 2005, 109, 11279.

37 C. N. R. Rao and K. P. Kalyanikutty, Acc. Chem. Res., 2008, 41, 489.

38 N. J. Halas, S. Lal, W. S. Chang, S. Link and P. Nordlander, Chem. Rev., 2011, 111, 3913.

39 R. E. LeRu and P. Etchegoin, Principles of Surface-Enhanced Raman Spectroscopy and Related Plasmonic effects, Elsevier, Amsterdam, 2009. 